\title{
A Note on the Time to Failure of a Two-Unit Parallel Redundant System with Deterioration on a Lattice
}

\author{
Tadashi Dohi \\ Department of Information Engineering, Hiroshima University, \\ 1-4-1 Kagamiyama, Higashi-Hiroshima 739-8527, Japan. \\ Corresponding author: dohi@ hiroshima-u.ac.jp \\ Junjun Zheng \\ Department of Information Science and Engineering, Ritsumeikan University, \\ 1-1-1 Nojihigashi Kusatsu 525-8577, Japan. \\ E-mail: jzheng@asl.cs.ritsumei.ac.jp \\ Hiroyuki Okamura \\ Department of Information Engineering, Hiroshima University, \\ 1-4-1 Kagamiyama, Higashi-Hiroshima 739-8527, Japan. \\ E-mail: okamu@hiroshima-u.ac.jp \\ (Received December 27, 2019; Accepted January 24, 2020)
}

\begin{abstract}
In this paper, we consider a two-unit parallel redundant system with deterioration on a lattice, where each unit has multistage deterioration levels, say, $n$ levels. The transition from one deterioration level to the subsequent level occurs following the well-known Marshall-Olkin bivariate exponential distribution. We derive the closed form of the Laplace transform of the time to system failure in the two-unit parallel redundant system with deterioration on $n \times n$ lattice without repair and simultaneous failure, as well as the simple system on $3 \times 3$ lattice.
\end{abstract}

Keywords- Two-unit parallel redundant system, Marshall-Olkin bivariate exponential distribution, Time to system failure, Laplace transform, Deterioration on a lattice.

\section{Introduction}

Two-unit parallel redundant systems play a central role to design actual redundant component systems in terms of fault-tolerance. In fact, a number of authors have paid their attentions to investigate the stochastic behaviour of redundant systems composed of two independent components (Borgerson and Freitas, 1975; Osaki and Nishio, 1980). On the other hand, a two-unit parallel redundant system with bivariate exponential lifetimes was first analyzed by Harris (1968), Osaki (1980). They applied the bivariate exponential distribution proposed by Marshall and Olkin (1967) to characterize the two-unit system with correlations. Sugasawa and Kaji (1981) and Goel et al. (1985) also took account of maintenance and inspection to the two-unit parallel system, respectively, for the two-unit parallel redundant systems. Sinha and Chaudhuri (1998) surveyed several results on the two-unit series systems based on the different definitions of bivariate exponential distributions. While the two-unit parallel redundant system is a rather standard system configuration, many authors (Ram and Singh, 2008; El-Said and El-Sherbeny, 2010; Ram et al., 2013; Singh et al., 2013; Ota et al., 2015) concerned to analyze different stochastic models as well.

In this paper, we consider a basic two-unit parallel redundant system with deterioration on a lattice. In the two-unit hot-standby parallel redundant system, each unit has multi-stage deterioration 
International Journal of Mathematical, Engineering and Management Sciences

Vol. 6, No. 1, 3-14, 2021

https://doi.org/10.33889/IJMEMS.2021.6.1.002

levels, say $n$ levels, and the transition from one deterioration level to the subsequent one occurs following an exponential distribution. More precisely, the deterioration time of the two-unit parallel system obeys a bivariate exponential distribution in the sense of Marshall and Olkin (1967), and the system failure time corresponds to the first passage time from the initial state $(i, j)=(0,0)$ to an absorbing state $(i, j)=(n-1, n-1)$ for respective units, where $i, j=0,1, \ldots, n-1$ and $n \geq$ 2. The present model under consideration is quite different from Harris (1968), Osaki (1980), and can be regarded as an abstract model for redundant structural systems in actual design.

The main purposes of this paper are to derive analytically (i) the Laplace transform of the time to failure in a simple two-unit parallel redundant system with deterioration on $3 \times 3$ lattice, and (ii) the Laplace transform of the time to failure in the two-unit parallel redundant system with deterioration on $n \times n$ lattice without repair and simultaneous failure. A numerical illustration is given to investigate the temporal behavior of the mean time to failure and its variance.

\section{Two-Unit Parallel Redundant System}

Let us consider a two-unit parallel redundant system with deterioration on a lattice. In the two-unit hot-standby parallel redundant system, each unit has multi-stage deterioration levels, say $n$ (= $2,3, \ldots)$ levels, and the transition from one deterioration level to the subsequent one occurs following an exponential distribution. More precisely, let $T_{1}$ and $T_{2}$ be the independent deterioration times for Unit 1 and Unit 2, respectively, and denote the non-negative random variables having the marginal distribution functions:

$$
\begin{aligned}
& F_{1}(x)=\operatorname{Pr}\left\{T_{1} \leq x\right\}=1-\exp \left\{-\lambda_{1} x\right\}, \quad x>0, \lambda_{1}>0, \\
& F_{2}(y)=\operatorname{Pr}\left\{T_{2} \leq y\right\}=1-\exp \left\{-\lambda_{2} y\right\}, \quad y>0, \lambda_{2}>0 .
\end{aligned}
$$

When both units may deteriorate simultaneously, on the other hand, let $T_{12}$ denote the simultaneous deterioration time for them having

$$
F_{12}(z)=\operatorname{Pr}\left\{T_{12} \leq z\right\}=1-\exp \left\{-\lambda_{12} z\right\}, \quad z>0, \lambda_{12}>0,
$$

In this case, the deterioration times for Unit 1 and Unit 2 taking account of the simultaneous deterioration are given by

$$
X=\min \left(T_{1}, T_{12}\right), \quad Y=\min \left(T_{2}, T_{12}\right),
$$

and the deterioration time distribution is described by the Marshall-Olkin bivariate exponential distribution (Marshall and Olkin, 1967):

$$
F(x, y)=\operatorname{Pr}\{X \leq x, Y \leq y\}=1-\exp \left\{-\lambda_{1} x-\lambda_{2} y-\lambda_{12} \max (x, y)\right\} .
$$

From Eq. (4) it is evident that $T_{1}, T_{2}$ and $T_{12}$ are independent exponentially distributed random variables. When one of units fails, the repair starts immediately and the repaired unit becomes as good as new. The repair times for Unit 1 and Unit 2 are independent from each other, and obey the common distribution functions $G_{1}(t)$ and $G_{2}(t)$, respectively. Hence, even if Unit 1 (2) fails, the system is operative during the period until an opponent Unit 2 (1) is operating, so that the state in which one of two units fails is a regeneration point, and the repair operation always starts at this point. On the other hand, the state in which both units fail is the non-regeneration point in this 
International Journal of Mathematical, Engineering and Management Sciences

Vol. 6, No. 1, 3-14, 2021

https://doi.org/10.33889/IJMEMS.2021.6.1.002

system (Osaki and Nishio, 1980). Figure 1 illustrates the configuration of the two-unit system on $n \times n$ lattice. In this system, each state is denoted by the pair $(x-y)$, where $x(=1,2)$ is the unit number and $y(=0,1, \ldots, n-1)$ is the deterioration level. The system failure time is defined by the first passage time from the initial state $(i, j)=(0,0)$ to an absorbing state $(i, j)=(n-1, n-$ 1) for respective units, with $i, j=0,1, \ldots, n-1$ and $n \geq 2$.

Define:

$q_{i j}(t):$ transition probability from state $i$ to state $j$ at time $t$, where $i, j=0,1, \ldots, n^{2}-1$,

$q_{i j}(s)$ Laplace transform of $q_{i j}(t)$, where $q_{i j}(s)=\int_{0}^{\infty} \exp \{-s t\} q_{i j}(t) d t$,

$P_{i}(t): \quad$ transition probability from state $i$ to the system failure,

$P_{i}(s): \quad$ Laplace transform of $P_{i}(t)$, where $P_{i}(s)=\int_{0}^{\infty} \exp \{-s t\} P_{i}(t) d t$.

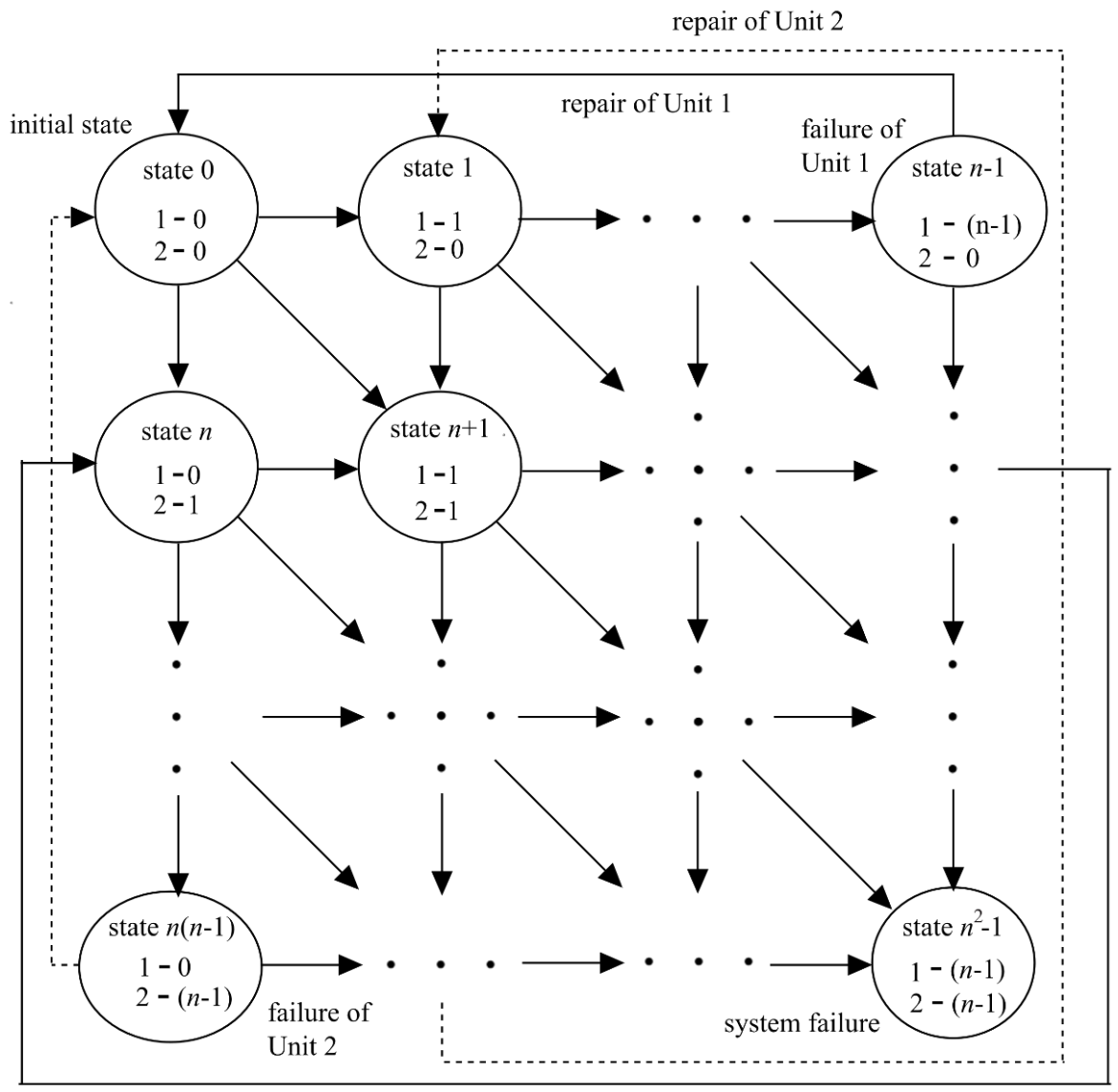

Figure 1. Configuration of the two-unit parallel redundant system with deterioration on $n \times n$ lattice. 
International Journal of Mathematical, Engineering and Management Sciences

Vol. 6, No. 1, 3-14, 2021

https://doi.org/10.33889/IJMEMS.2021.6.1.002

From the well-known Markovian analysis, it is straightforward to see that

$$
P_{i}(t)=\sum_{j} \int_{0}^{t} P_{j}(t-x) q_{i j}(x) d x
$$

Taking the Laplace transform of both sides in Eq. (6), we have

$$
P_{i}(s)=\int_{0}^{\infty} \exp \{-s t\} P_{i}(t) d t=\sum_{j} q_{i j}(s) P_{j}(s) .
$$

The problem is reduced to derive the Laplace transform of the time to failure, $P_{0}(s)$. Since $q_{i j}(t), i, j=0,1, \ldots, n^{2}-1$, are the exponential transition probabilities, it would be possible to calculate $P_{i}(t)$ numerically even for relatively large $n$. In the following sections, we focus on the analytically tractable cases and derive some useful results.

\section{Special Case with $n=3$}

As one of the simplest cases, we consider a two-unit parallel redundant system with deterioration on $3 \times 3$ lattice. This simple situation frequently occurs in many examples. For instance, Huang et al. (1995) and Garg et al. (1995) considered an AT \& T billing application and described the software degradation behavior called software aging by a continuous-time Markov chain and a Markov regenerative process, respectively. In their modeling, it is assumed that the system failure occurs through one degradation level called failure probable state. So, if the software system is a one-unit system, the time to failure distribution is given by the Stieltjes convolution of two nonidentical exponential distributions, which is the hypo-exponential distribution. Rinsaka and Dohi (2005) performed the behavioral analysis of a two-unit fault-tolerant software system with rejuvenation and formulated the system availability, where the number of degradation levels for each unit is two. In this way, we note that the environmental diversity in software systems is based on the redundant configuration by either an identical replica of software or a non-identical hot standby system (Trivedi and Bobbio, 2017).

In what follows, we consider the two-unit parallel redundant software system with deterioration on $3 \times 3$ lattice, which is illustrated in Figure 2. Define the following state $i(=0,1, \ldots, 8)$ :

State 0 (4): Both units are operating (deteriorating),

State 1 (3): Unit 1 (2) is deteriorating, but Unit 2 (1) is operating,

State 2 (6): Unit 1 (2) fails, but Unit 2 (1) is operating,

State 5 (7): Unit 1 (2) fails, but Unit 2 (1) is deteriorating,

State 8: $\quad$ Both units fail and the system failure occurs.

It can be found that the regeneration points are States 2, 5, 6 and 7, and that State 8 is a nonregeneration point.

Next, we derive the probability distribution function of the time to system failure. Let $q_{01}(t)$ be the transition probability at time $t$ from State 0 to State 1 without visiting both State 3 and State 4 . Then, we have 
International Journal of Mathematical, Engineering and Management Sciences

Vol. 6, No. 1, 3-14, 2021

https://doi.org/10.33889/IJMEMS.2021.6.1.002

$$
q_{01}(t)=\exp \left\{-\lambda_{2} t\right\} \exp \left\{-\lambda_{12} t\right\} \cdot \frac{d}{d t}\left(1-\exp \left\{-\lambda_{1} t\right\}\right)=\lambda_{1} \exp \left\{-\left(\lambda_{1}+\lambda_{2}+\lambda_{12}\right) t\right\}
$$

The Laplace transform of $q_{01}(t)$ is given by

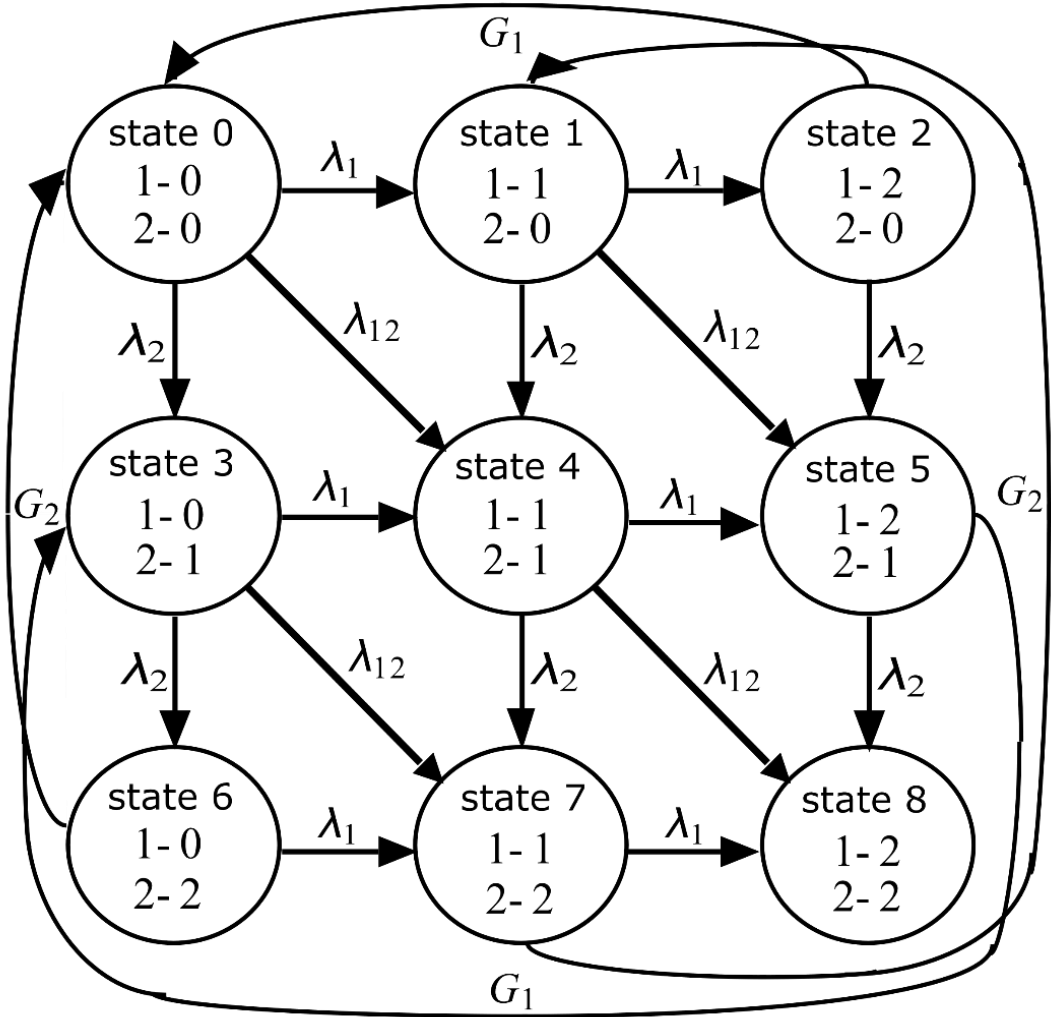

Figure 2. Configuration of the two-unit parallel redundant system with deterioration on $3 \times 3$ lattice.

$$
q_{01}(s)=\int_{0}^{\infty} \exp \{-s t\} \lambda_{1} \exp \left\{-\left(\lambda_{1}+\lambda_{2}+\lambda_{12}\right) t\right\} d t=\lambda_{1} /(s+\lambda)
$$

where, $\lambda=\lambda_{1}+\lambda_{2}+\lambda_{12}$. Similarly, we obtain

$$
\begin{aligned}
& q_{12}(s)=q_{34}(s)=q_{45}(s)=q_{01}(s)=\int_{0}^{\infty} \exp \{-s t\} \lambda_{1} \exp \{-\lambda t\} d t=\lambda_{1} /(s+\lambda), \\
& q_{03}(s)=q_{36}(s)=q_{14}(s)=q_{47}(s)=\int_{0}^{\infty} \exp \{-s t\} \lambda_{2} \exp \{-\lambda t\} d t=\lambda_{2} /(s+\lambda), \\
& q_{04}(s)=q_{15}(s)=q_{37}(s)=q_{48}(s)=\int_{0}^{\infty} \exp \{-s t\} \lambda_{12} \exp \{-\lambda t\} d t=\lambda_{12} /(s+\lambda),
\end{aligned}
$$


International Journal of Mathematical, Engineering and Management Sciences

Vol. 6, No. 1, 3-14, 2021

https://doi.org/10.33889/IJMEMS.2021.6.1.002

$$
\begin{gathered}
q_{20}(s)=q_{53}(s)=\int_{0}^{\infty} \exp \{-s t\} \exp \left\{-\lambda_{2} t\right\} d G_{1}(t)=g_{1}\left(s+\lambda_{2}\right), \\
\begin{aligned}
q_{25}(s)=q_{58}(s & =\int_{0}^{\infty} \exp \{-s t\} \lambda_{212} \exp \left\{-\lambda_{212} t\right\} \bar{G}_{1}(t) d t \\
& =\lambda_{212}\left\{1-g_{1}\left(s+\lambda_{212}\right)\right\} /\left(s+\lambda_{212}\right),
\end{aligned} \\
\begin{aligned}
q_{60}(s)=q_{71}(s) & =\int_{0}^{\infty} \exp \{-s t\} \exp \left\{-\lambda_{1} t\right\} d G_{2}(t)=g_{2}\left(s+\lambda_{1}\right), \\
q_{67}(s)=q_{78}(s & =\int_{0}^{\infty} \exp \{-s t\} \lambda_{112} \exp \left\{-\lambda_{112} t\right\} \bar{G}_{2}(t) d t \\
& =\lambda_{112}\left\{1-g_{2}\left(s+\lambda_{112}\right)\right\} /\left(s+\lambda_{112}\right),
\end{aligned}
\end{gathered}
$$

where, in general, $\bar{\varphi}(\cdot)=1-\varphi(\cdot), \lambda_{212}=\lambda_{2}+\lambda_{12}, \lambda_{112}=\lambda_{1}+\lambda_{12}$ and

$$
g_{i}(s)=\int_{0}^{\infty} e^{-s t} d G_{i}(t), \quad i=1,2
$$

From Eqs. (7) to (17), we obtain the following result.

Theorem 3.1: In the two-unit parallel redundant system with deterioration on $3 \times 3$ lattice, the Laplace transform of the time to system failure is given by

$$
P_{0}(s)=q_{01}(s) P_{1}(s)+q_{03}(s) P_{3}(s)+q_{04}(s) P_{4}(s),
$$

where,

$$
\begin{aligned}
& P_{1}(s)=q_{12}(s) P_{2}(s)+q_{14}(s) P_{4}(s)+q_{15}(s) P_{5}(s) \\
& P_{2}(s)=q_{20}(s) P_{0}(s)+q_{25}(s) P_{5}(s) \\
& P_{3}(s)=q_{34}(s) P_{4}(s)+q_{36}(s) P_{6}(s)+q_{37}(s) P_{7}(s) \\
& P_{4}(s)=q_{45}(s) P_{5}(s)+q_{47}(s) P_{7}(s)+q_{48}(s) \\
& P_{5}(s)=q_{58}(s)+q_{53}(s) P_{3}(s) \\
& P_{6}(s)=q_{60}(s) P_{0}(s)+q_{67}(s) P_{7}(s) \\
& P_{7}(s)=q_{78}(s)+q_{71}(s) P_{1}(s)
\end{aligned}
$$

From Theorem 3.1, we can calculate the time to system failure distribution for the general repair time distributions $G_{i}(t)(i=1,2)$. Looking at Eq. (7), it seems difficult to derive an explicit form of $P_{0}(s)$ for $n \geq 4$. In the next section, we simplify the assumptions on the repair and simultaneous failure. 
International Journal of Mathematical, Engineering and Management Sciences

Vol. 6, No. 1, 3-14, 2021

https://doi.org/10.33889/IJMEMS.2021.6.1.002

\section{Special Case without Repair and Simultaneous Failure}

Hereafter, we consider much simpler cases where both repair and simultaneous failure do not occur, but, instead, the lattice size is general, say, $n \times n$ lattice. That is, we suppose that $\lambda_{1}=\lambda_{2}, \lambda_{12}=$ 0 and $G_{1}(t)=G_{2}(t)=0$. In the example on software aging (Garg et al., 1995; Huang et al., 1995), the assumptions of $\lambda_{1}=\lambda_{2}$ and $\lambda_{12}=0$ do not loss the generality, because identical replica of one software system puts as a hot stand-by unit. To simplify the notation, we define $a(s) \equiv q_{01}(s)=$ $q_{12}(s)=\cdots=q_{n-2 n-1}(s)$ and $c(s) \equiv q_{n-12 n-1}(s)=q_{2 n-13 n-1}(s)=\cdots=q_{n^{2}-n-1 n^{2}-1}(s)$.

First consider the case of $2 \times 2$ lattice. From a few algebraic manipulations, it is straightforward to get

$$
\begin{aligned}
& a(s)=q_{01}(s)=q_{02}(s)=\int_{0}^{\infty} e^{-s t} \lambda_{1} e^{-\lambda_{1} t} e^{-\lambda_{1} t}, \\
& c(s)=q_{13}(s)=q_{23}(s)=\int_{0}^{\infty} e^{-s t} \lambda_{1} e^{-\lambda_{1} t} .
\end{aligned}
$$

Hence, the Laplace transform of the time to failure in the two-unit parallel redundant system deteriorating on $2 \times 2$ lattice is given by

$$
P_{0}(s)=2 a(s) c(s) \text {, }
$$

where,

$$
\begin{aligned}
& P_{0}(s)=q_{01}(s) P_{1}(s)+q_{02}(s) P_{2}(s)=a(s) P_{1}(s)+a(s) P_{2}(s) \\
& P_{1}(s)=q_{13}(s)=c(s) \\
& P_{2}(s)=q_{23}(s)=c(s) .
\end{aligned}
$$

In the case with $3 \times 3$ lattice, from Eqs. (18) to (25), we immediately obtain

$$
P_{0}(s)=4 a(s)^{3} c(s)+2 a(s)^{2} c(s)^{2},
$$

where,

$$
\begin{aligned}
& a(s)=q_{01}(s)=q_{02}(s)=q_{12}(s)=q_{36}(s)=q_{14}(s)=q_{47}(s)=q_{34}(s)=q_{45}(s), \\
& c(s)=q_{25}(s)=q_{58}(s)=q_{67}(s)=q_{78}(s), \\
& P_{0}(s)=q_{01}(s) P_{1}(s)+q_{03}(s) P_{3}(s)=a(s) P_{1}(s)+a(s) P_{3}(s), \\
& P_{1}(s)=q_{12}(s) P_{2}(s)+q_{14}(s) P_{4}(s)=a(s) P_{2}(s)+a(s) P_{4}(s), \\
& P_{2}(s)=q_{25}(s) P_{5}(s)=c(s) P_{5}(s), \\
& P_{3}(s)=q_{34}(s) P_{4}(s)+q_{36}(s) P_{3}(s)=a(s) P_{1}(s)+a(s) P_{3}(s), \\
& P_{4}(s)=q_{45}(s) P_{5}(s)+q_{47}(s) P_{7}(s)=a(s) P_{5}(s)+a(s) P_{7}(s),
\end{aligned}
$$


International Journal of Mathematical, Engineering and Management Sciences

Vol. 6, No. 1, 3-14, 2021

https://doi.org/10.33889/IJMEMS.2021.6.1.002

$$
\begin{aligned}
& P_{5}(s)=q_{58}(s)=c(s), \\
& P_{6}(s)=q_{67}(s) P_{7}(s)=c(s) P_{7}(s), \\
& P_{7}(s)=q_{78}(s)=c(s) .
\end{aligned}
$$

Further, we derive the Laplace transform of the time to failure in the two-unit parallel redundant system with deterioration on $4 \times 4$ lattice as follows.

$$
P_{0}(s)=12 a(s)^{5} c+6 a(s)^{4} c(s)^{3}+2 a(s)^{3} c(s)^{3},
$$

where,

$$
\begin{aligned}
& a(s)=q_{01}(s)=q_{12}(s)=q_{23}(s)=q_{45}(s)=q_{56}(s)=q_{67}(s)=q_{89}(s)=q_{910}(s) \\
& =q_{1011}(s)=q_{04}(s)=q_{48}(s)=q_{812}(s)=q_{15}(s)=q_{59}(s) \\
& =q_{913}(s)=q_{26}(s)=q_{610}(s)=q_{1014}(s) \text {, } \\
& c(s)=q_{37}(s)=q_{711}(s)=q_{1115}(s)=q_{1213}(s)=q_{1314}(s)=q_{1415}(s) \text {, } \\
& P_{0}(s)=q_{01}(s) P_{1}(s)+q_{04}(s) P_{4}(s)=a(s) P_{1}(s)+a(s) P_{4}(s) \text {, } \\
& P_{1}(s)=q_{12}(s) P_{2}(s)+q_{15}(s) P_{5}(s)=a(s) P_{2}(s)+a(s) P_{5}(s) \text {, } \\
& P_{2}(s)=q_{23}(s) P_{3}(s)+q_{26}(s) P_{6}(s)=a(s) P_{3}(s)+a(s) P_{6}(s), \\
& P_{3}(s)=q_{37}(s) P_{7}(s)=c(s) P_{7}(s) \text {, } \\
& P_{4}(s)=q_{45}(s) P_{5}(s)+q_{48}(s) P_{8}(s)=a(s) P_{5}(s)+a(s) P_{8}(s) \text {, } \\
& P_{5}(s)=q_{56}(s) P_{6}(s)+q_{59}(s) P_{9}(s)=a(s) P_{6}(s)+a(s) P_{9}(s) \text {, } \\
& P_{6}(s)=q_{67}(s) P_{7}(s)+q_{610}(s) P_{10}(s)=a(s) P_{7}(s)+a(s) P_{10}(s), \\
& P_{7}(s)=q_{711}(s) P_{11}(s)=c(s) P_{11}(s) \text {, } \\
& P_{8}(s)=q_{89}(s) P_{9}(s)+q_{812}(s) P_{12}(s)=a(s) P_{9}(s)+a(s) P_{12}(s) \text {, } \\
& P_{9}(s)=q_{910}(s) P_{10}(s)+q_{913}(s) P_{13}(s)=a(s) P_{10}(s)+a(s) P_{13}(s) \text {, } \\
& P_{10}(s)=q_{1011}(s) P_{11}(s)+q_{1014}(s) P_{14}(s)=a(s) P_{11}(s)+a(s) P_{14}(s) \text {, } \\
& P_{11}(s)=q_{1115}(s)=c(s) \text {, } \\
& P_{12}(s)=q_{1213}(s) P_{13}(s)=c(s) P_{13}(s) \text {, } \\
& P_{13}(s)=q_{1314}(s) P_{14}(s)=c(s) P_{14}(s) \text {, } \\
& P_{14}(s)=q_{1415}(s)=c(s) \text {. }
\end{aligned}
$$

Finally, from the inductive argument, we derive the main results of this paper in the following:

Theorem 4.1 In the two-unit parallel redundant system deteriorating on $n \times n$ lattice without repair and simultaneous failure, the Laplace transform of the time to system failure is by 
International Journal of Mathematical, Engineering and Management Sciences

Vol. 6, No. 1, 3-14, 2021

https://doi.org/10.33889/IJMEMS.2021.6.1.002

$$
P_{0}(s)=2 a(s) \sum_{k=1}^{n-1}\left(\begin{array}{c}
2 n-k-3 \\
n-2
\end{array}\right) a(s)^{2 n-k-3} c(s)^{k}
$$

where, $n \geq 2, a(s)=\lambda_{1} /\left(s+2 \lambda_{1}\right)$ and $c(s)=\lambda_{1} /\left(s+\lambda_{1}\right)$.

Corollary 4.2 In the two-unit parallel redundant system deteriorating on $n \times(n-1)$ lattice without repair and simultaneous failure, the Laplace transform of the time to failure is $P_{0}(s) /(2 a(s))$, where $P_{0}(s)$ is given in Eq. (61).

Corollary 4.3 In the two-unit parallel redundant system deteriorating on $n \times n$ lattice, the mean and variance of the time to failure are given by

$$
\begin{gathered}
\mathrm{E}[T]=\frac{1}{\lambda_{1}} \sum_{k=1}^{n-1}\left(\begin{array}{c}
2 n-k-3 \\
n-2
\end{array}\right)\left\{2^{-2 n+k+2}(2 n-k-2)+2^{-2 n+k+3} k\right\}, \\
\operatorname{Var}[T]=\frac{1}{\lambda_{1}^{2}} \sum_{k=1}^{n-1}\left(\begin{array}{c}
2 n-k-3 \\
n-2
\end{array}\right)\left\{(2 n-k-2)(2 n-k-1) 2^{-2 n+k}+k(2 n-k\right. \\
\left.-2) 2^{-2 n+k+2}+k(k+1) 2^{-2 n+k+2}\right\}-E[T]^{2},
\end{gathered}
$$

respectively.

Corollary 4.4 In the two-unit parallel redundant system deteriorating on $n \times(n-1)$ lattice, the mean and variance of the time to failure are given by

$$
\begin{gathered}
\mathrm{E}[T]=\frac{1}{\lambda_{1}} \sum_{k=1}^{n-1}\left(\begin{array}{c}
2 n-k-3 \\
n-2
\end{array}\right)\left\{2^{-2 n+k+1}(2 n-k-3)+2^{-2 n+k+2} k\right\}, \\
\operatorname{Var}[T]=\frac{1}{\lambda_{1}{ }^{2}} \sum_{k=1}^{n-1}\left(\begin{array}{c}
2 n-k-3 \\
n-2
\end{array}\right)\left\{(2 n-k-3)(2 n-k-2) 2^{-2 n+k+1}+k(2 n-k\right. \\
\left.-3) 2^{-2 n+k+3}+k(k+1) 2^{-2 n+k+3}\right\}-E[T]^{2},
\end{gathered}
$$

respectively.

Corollary 4.2 and Corollary 4.4 are the direct applications of Theorem 4.1 and Corollary 4.3, respectively, and are useful to analyze an asymmetric lattice structure on deterioration.

We investigate numerically the temporal behavior of the mean time to failure and its variance. In Figures 3 and 4, we plot the mean time to failure and the variance with respect to the number of deterioration levels on $n \times n$ lattice. For an arbitrary $\lambda_{1}(>0)$, the mean time to failure increases gradually as $n$ increases, where the increment between $n-1$ and $n$ approaches to $1 / \lambda_{1}$ from a larger value. The increasing rate of the variance is higher than the mean time to failure but the increment is somewhat milder. 
International Journal of Mathematical, Engineering and Management Sciences

Vol. 6, No. 1, 3-14, 2021

https://doi.org/10.33889/IJMEMS.2021.6.1.002

\section{Mean Failure Time}

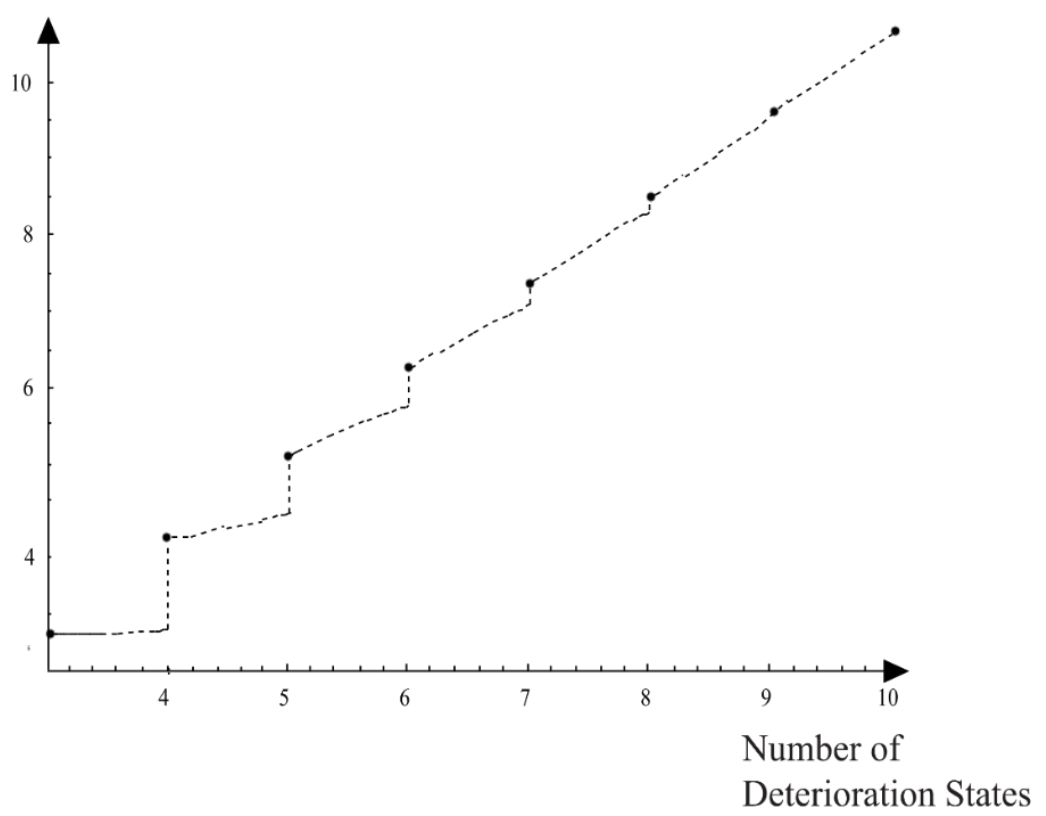

Figure 3. Mean failure time in the two-unit parallel redundant system with deterioration on $n \times n$ lattice.

Variance of Failure Time

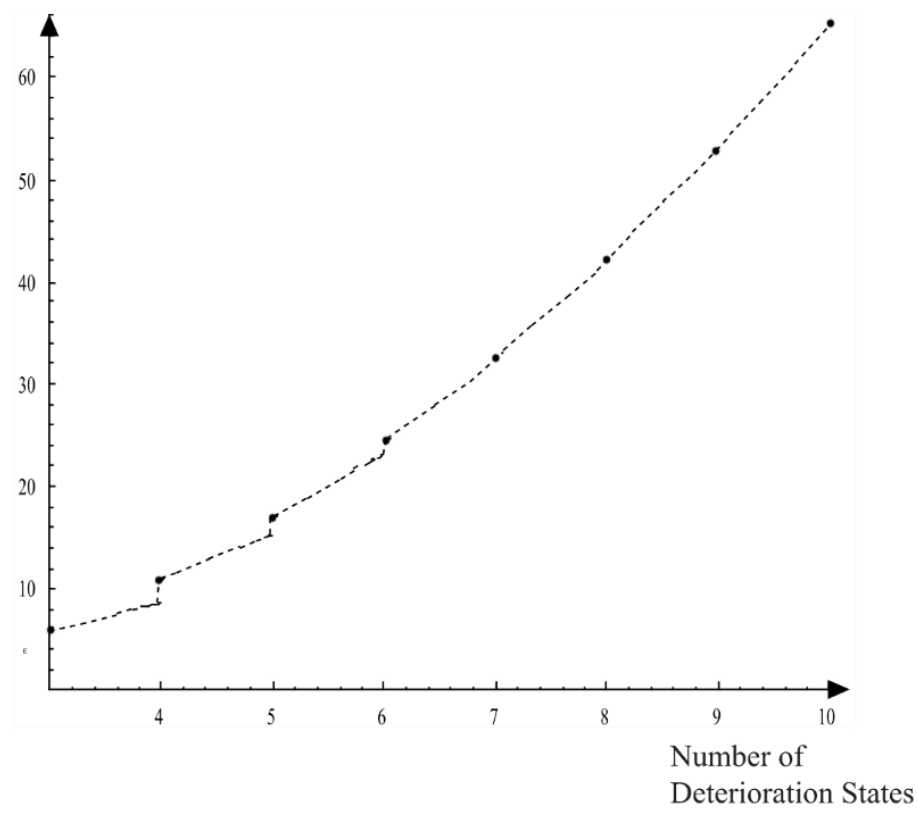

Figure 4. Variance of failure time in the two-unit parallel redundant system with deterioration on $n \times n$ lattice. 
International Journal of Mathematical, Engineering and Management Sciences

Vol. 6, No. 1, 3-14, 2021

https://doi.org/10.33889/IJMEMS.2021.6.1.002

\section{Conclusions}

In this paper, we have considered a two-unit parallel redundant system with deterioration on a lattice, where each unit has multi-stage deterioration levels under the assumption that the transition from one deterioration level to the subsequent level occurred following the well-known MarshallOlkin bivariate exponential distribution. We have derived the closed form of the Laplace transform of the time to failure in the two-unit parallel redundant system with deterioration on $n \times n$ lattice without repair and simultaneous failure, as well as the simple system on $3 \times 3$ lattice. Our explicit results in this paper can be easily applied to a general case where the degradation time of both units follows a Marshall-Olkin type bivariate distribution with the common marginal distributions.

Once the Laplace transform was derived, the Laplace inversion technique is applied to calculate the time to failure distribution numerically. However, the Laplace inversion transform is still instable in computation, and does not always provide feasible solutions. In future, we will propose any approximation scheme to compute the time to system failure distribution without the inversion technique.

\section{Conflict of Interest}

The authors confirm that there is no conflict of interest to declare for this publication.

\section{Acknowledgments}

Dedicated to Professor S. B. Singh, Department of Mathematics, Statistics and Computer Science, G. B. Pant University of Agriculture and Technology, Pantnagar, India on his $52^{\text {nd }}$ birthday in recognition of his contributions and leadership of research in reliability and maintenance in India.

\section{References}

Borgerson, B.R., \& Freitas, R.F. (1975). A reliability model for gracefully degrading and standby-sparing systems. IEEE Transactions on Computers, C-24(5), 517-525.

El-Said, K.M., \& El-Sherbeny, M.S. (2010). Stochastic analysis of a two-unit cold standby system with twostage repair and waiting time. Sankhya B, 72,1-10. doi:10.1007/s13571-010-0001-9.

Garg, S., Puliafito, A., Telek, M., \& Trivedi, K.S. (1995). Analysis of software rejuvenation using Markov regenerative stochastic Petri net. In Proceedings of the $6^{\text {th }}$ International Symposium on Software Reliability Engineering (ISSRE'95) (pp. 180-187). IEEE. Toulouse, France.

Goel, L.R., Gupta, R., \& Singh, S.K. (1985). Availability analysis of a two-unit (dissimilar) parallel system with inspection and bivariate exponential life times. Microelectronics and Reliability, 25(1), 77-80.

Harris, R. (1968). Reliability applications of a bivariate exponential distribution. Operations Research, 16(1), 18-27.

Huang, Y., Kintala, C., Kolettis, N., \& Funton, N.D. (1995). Software rejuvenation: analysis, module and applications. In Proceedings of the 25th IEEE International Symposium on Fault Tolerant Computing (FTC'95) (pp. 381-390). IEEE. Pasadena, CA, USA.

Marshall, A.W., \& Olkin, I. (1967). A multivariate exponential distribution. Journal of the American Statistical Association, 62(317), 30-44.

Osaki, S. (1980). A two-unit parallel redundant system with bivariate exponential lifetimes. Microelectronics and Reliability, 20(4), 521-523. 
International Journal of Mathematical, Engineering and Management Sciences

Vol. 6, No. 1, 3-14, 2021

https://doi.org/10.33889/IJMEMS.2021.6.1.002

Osaki, S., \& Nishio, T. (1980). Reliability Evaluation of Some Fault-Tolerant Computer Architecture. Lecture Notes in Computer Science, 9, Springer-Verlag, Berlin.

Ota, S., Kageyama, T., \& Kimura, M. (2015). Improvement of reliability evaluation for 2-unit parallel system with cascading failures by using maximal copula. IEICE Transactions on Fundamentals of Electronics, Communications and Computer Sciences, EA-98(10), 2096-2100.

Ram, M., \& Singh, S.B. (2008). Availability and cost analysis of a parallel redundant complex system with two types of failure under preemptive-resume repair discipline using Gumbel-Hougaard family copula in repair. International Journal of Reliability, Quality and Safety Engineering, 15(4), 341-365.

Ram, M., Singh, S.B., \& Singh, V.V. (2013). Stochastic analysis of a standby system with waiting repair strategy. IEEE Transactions on Systems, Man, and Cybernetics: Systems, 43(3), 698-707.

Rinsaka, K., \& Dohi, T. (2005). Behavioral analysis of fault-torellant software systems with rejuvenation. IEICE Transactions on Information and Systems (D), ED-88(12), 2681-2690.

Singh, V.V., Singh, S.B., Ram, M., \& Goel, C.K. (2013). Availability, MTTF and cost analysis of a system having two units in series configuration with controller. International Journal of System Assurance Engineering and Management, 4(4), 341-352.

Sinha, B.K., \& Chaudhuri, G. (1998). Complete confounding in a two-component series system: status of bivariate exponentials. In: Basu, A.P., Basu, S.K., \& Mukhopadhyay, S. (eds) Frontiers in Reliability. World Scientific, Singapore, pp. 361-366.

Sugasawa, Y., \& Kaji, I. (1981). Light maintenance for a two-unit parallel redundant system with bivariate exponential lifetimes. Microelectronics and Reliability, 21(5), 661-670.

Trivedi, K.S., \& Bobbio, A. (2017). Reliability and Availability Engineering, Modeling, Analysis, and Applications. Cambridge University Press. U.K. 